\title{
A Subspace Identification Algorithm based on Stochastic Realization with Exogenous Inputs
}

\author{
Tohru Katayama and Shogo Omori
}

\author{
Department of Applied Mathematics and Physics \\ Graduate School of Informatics, Kyoto University, Kyoto 606-01, Japan \\ e-mail: katayama@kuamp.kyoto-u.ac.jp
}

\section{Abstract}

In the earlier paper (Katayama and Picci, 1997), we have developed a stochastic realization method for a linear discrete-time system with an exogenous input. In this paper we present a stochastic subspace identification algorithm. We also compare the present algorithm and the CLS algorithm due to Peternell et al.(1996).

Keywords: Stochastic realization; Canonical correlation analysis; Subspace system identification.

\section{Introduction}

Subspace identification methods have received much interest in the past years; see Larimore (1990), Moonen and Vandewalle $(1989,1990)$, Van Overschee and De Moor $(1993,1994,1996)$, Verhaegen and Dewilde (1992), Verhaegen (1994), Viberg (1995), to name but a few. Moreover, relations among existing subspace identification methods have been discussed by Van Overschee and De Moor (1996). Subspace methods involve geometric operations on subspaces spanned by the column or row vectors of certain block Hankel matrices formed by the input-output data. These operations are performed numerically in a reliable way based on the singular value decomposition (SVD) and QR decomposition (Golub and Van Loan, 1989); a main advantage being that the difficult model selection problem inherent in the classical parameter optimization approaches to MIMO identification is completely avoided.

Recently Lindquist and Picci (1996) have interpreted subspace identification of state-space models from the point of view of realization theory. Picci and Katayama (1996) have shown the relevance of stochastic realization to subspace identification of state-space systems with exogenous inputs. Also, we have derived a stochastic realization method for the system excited by an exogenous input based on oblique projection and the canonical correlation analysis (Katayama and Picci, 1997).
In this paper, we present a stochastic subspace identification algorithm based on the result of Katayama and Picci (1997). We also compare the present algorithm and the CLS algorithm due to Peternell et al.(1996).

\section{Stochastic Realization Method}

Consider a discrete-time stochastic linear system with the $m \times 1$ input vector $u(t)$ and the $p \times 1$ output vector $y(t)$. It is assumed that $\{u(t), y(t), t=0, \pm 1, \cdots\}$ are jointly wide sense stationary processes with zero mean and finite covariance matrices.

Let $t$ be the present time and $k$ a positive integer. We then define the stacked vectors of past and future inputs and stacked vectors of past and future of outputs as

$$
\mathbf{u}_{-}(t):=\left[\begin{array}{c}
u(t-1) \\
u(t-2) \\
\vdots
\end{array}\right], \quad \mathbf{u}_{+}(t):=\left[\begin{array}{c}
u(t) \\
u(t+1) \\
\vdots \\
u(t+k-1)
\end{array}\right]
$$

and

$$
\mathbf{y}_{-}(t):=\left[\begin{array}{c}
y(t-1) \\
y(t-2) \\
\vdots
\end{array}\right], \quad \mathbf{y}_{+}(t):=\left[\begin{array}{c}
y(t) \\
y(t+1) \\
\vdots \\
y(t+k-1)
\end{array}\right]
$$

where the present is conventionally included in the future. For notational simplicity, we also define

$$
\begin{aligned}
\mathbf{p}(t) & :=\left[\begin{array}{l}
\mathbf{u}_{-}(t) \\
\mathbf{y}_{-}(t)
\end{array}\right] \quad[\infty \times 1 \text { past observations }] \\
\mathbf{f}(t) & :=\mathbf{y}_{+}(t) \quad[k p \times 1 \text { future outputs }] \\
\mathbf{u}(t) & :=\mathbf{u}_{+}(t) \quad[k m \times 1 \text { future inputs }]
\end{aligned}
$$


Suppose that $\mathbf{a}, \mathbf{b}$ are random vectors with zero mean and finite covariance matrices. Then the orthogonal projection of $\mathbf{b}$ onto $\mathbf{a}$ is given by

$$
\mathbf{b} / \mathbf{a}=E\left(\mathbf{b a}^{T}\right) E\left(\mathbf{a a}^{T}\right)^{\dagger} \mathbf{a}
$$

where $E(\cdot)$ denotes the mathematical expectation, and $(\cdot)^{\dagger}$ is the pseudo-inverse (Golub and Van Loan, 1989). Let $\mathrm{a}^{\perp}$ be the orthogonal complement of the space generated by $\mathbf{a}$. Then the orthogonal projection of $\mathbf{b}$ onto $\mathbf{a}^{\perp}$ is defined by $\mathbf{b} / \mathbf{a}^{\perp}:=\mathbf{b}-(\mathbf{b} / \mathbf{a})$. Also, the covariance matrix of $\mathbf{a}$ and $\mathbf{b}$ is defined by $\Sigma_{a b}:=E\left\{\mathbf{a b}^{T}\right\}$.

\section{The Optimal Predictor}

Theorem 1. Suppose that $\mathbf{p}(t) \cap \mathbf{u}(t)=0$. Then the optimal LS predictor $\hat{\mathbf{f}}(t)$, of the future output vector $\mathbf{f}(t)$, based on the past input-output data $\mathbf{p}(t)$ and future inputs $\mathbf{u}(t)$, is given by

$$
\hat{\mathbf{f}}(t)=(\mathbf{f}(t) / \mathbf{p}(t) \vee \mathbf{u}(t))=\Pi \mathbf{p}(t)+\Phi \mathbf{u}(t)
$$

where $\Pi, \Phi$ are given by

$$
\begin{gathered}
{\left[\begin{array}{ll}
\Pi \Phi & \Phi
\end{array}=\left[\begin{array}{ll}
E\left(\mathbf{f p}^{T}\right) & E\left(\mathbf{f u}^{T}\right)
\end{array}\right]\left[\begin{array}{ll}
E\left(\mathbf{p p}^{T}\right) & E\left(\mathbf{p u}^{T}\right) \\
E\left(\mathbf{u p}^{T}\right) & E\left(\mathbf{u u}^{T}\right)
\end{array}\right]^{-1}\right.} \\
=\left[\begin{array}{ll}
\Sigma_{f p} & \Sigma_{f u}
\end{array}\right]\left[\begin{array}{cc}
\Sigma_{p p} & \Sigma_{p u} \\
\Sigma_{u p} & \Sigma_{u u}
\end{array}\right]^{-1}
\end{gathered}
$$

It can be also shown that the operators II and $\Phi$ satisfy the discrete Wiener-Hopf type equations

$$
\Pi \Sigma_{p p / u}=\Sigma_{f p / u}, \quad \Phi \Sigma_{u u / p}=\Sigma_{f u / p}
$$

where $\Sigma_{p p / u}, \Sigma_{u u / p}$ are the conditional covariance operators of the past vector $\mathbf{p}(t)$ given $\mathbf{u}(t)$ and of the future input $\mathbf{u}(t)$ given the past $\mathbf{p}(t)$, and are defined by

$$
\Sigma_{a b / c}:=E\left\{\left(\mathbf{a} / \mathbf{c}^{\perp}\right)\left(\mathbf{b} / \mathrm{c}^{\perp}\right)^{T}\right\}=\Sigma_{a b}-\Sigma_{a c} \Sigma_{c c}^{-1} \Sigma_{c b}
$$

It should be noted that $\Pi p(t)$ is the oblique projection of the future $\mathbf{f}(t)$ onto the past $\mathbf{p}(t)$ along the future $\mathbf{u}(t)$ and $\Phi \mathbf{u}(t)$ is the oblique projection of the future $\mathbf{f}(t)$ onto $\mathbf{u}(t)$ along $\mathbf{p}(t)$.

Theorem 2. Let $\zeta(t):=\left[\begin{array}{l}y(t) \\ u(t)\end{array}\right]$ be the jointly stationary regular full rank process. Suppose that $\zeta(t)$ is feedback-free. Then $\Phi$ is given by

$$
\Phi=\left[\begin{array}{cccc}
g_{0} & & & \\
g_{1} & g_{0} & & \\
\vdots & \vdots & \ddots & \\
g_{k-1} & g_{k-2} & \cdots & g_{0}
\end{array}\right]
$$

where $\left\{g_{0}, g_{1}, \cdots\right\}$ are impulse response matrices from the input $u(t)$ to the output $y(t)$. Thus $\Phi$ is block lowertriangular, so that it is a causal operator.

\section{State Vector}

Let $\operatorname{rank} \Sigma_{f p / u}=n$, and suppose that $k>n$. Consider the Cholesky factorizations $\Sigma_{p p / u}=L_{p} L_{p}^{T}$ and $\Sigma_{f f / u}=$ $L_{f} L_{f}^{T}$. Define

$$
\varepsilon_{+}(t):=L_{f}^{-1}\left(\mathbf{f} / \mathbf{u}^{\perp}\right)(t), \quad \varepsilon_{-}(t):=L_{p}^{-1}\left(\mathbf{p} / \mathbf{u}^{\perp}\right)(t)
$$

It then follows that

$$
E\left\{\varepsilon_{+}(t) \varepsilon_{-}^{T}(t)\right\}=L_{f}^{-1} \Sigma_{f p / u} L_{p}^{-T}
$$

Suppose that the SVD of the normalized block Hankel matrix $L_{f}^{-1} \Sigma_{f p / u} L_{p}^{-T}$ be given by

$$
L_{f}^{-1} \Sigma_{f p / u} L_{p}^{-T}=U \tilde{\Sigma} V^{T}
$$

where $U^{T} U=I_{n}, \quad V^{T} V=I_{n}$ and $\tilde{\Sigma}=\operatorname{diag}\left(\tilde{\sigma}_{1}, \cdots, \tilde{\sigma}_{n}\right)$ is a diagonal matrix with nonzero singular values $(1 \geq$ $\tilde{\sigma}_{1} \geq \cdots \geq \tilde{\sigma}_{n}>0$ ). We see that $\sigma_{i}^{\prime} s$ are the canonical correlation coefficients between the random vectors $\left(\mathbf{f} / \mathbf{u}^{\perp}\right)(t)$ and $\left(\mathbf{p} / \mathbf{u}^{\perp}\right)(t)$.

For the SVD of (5), we define the extended observability and controllability matrices as

$$
\mathcal{O}:=L_{f} U \tilde{\Sigma}^{1 / 2}, \quad \mathcal{C}:=\tilde{\Sigma}^{1 / 2} V^{T} L_{p}^{T}
$$

where $\operatorname{rank} \mathcal{O}=\operatorname{rank} \mathcal{C}=n$. Then the block Hankel matrix $\Sigma_{f p / u}$ has a well-known decomposition

$$
\Sigma_{f p / u}=\mathcal{O C}
$$

Since $\Pi=\Sigma_{f p / u} \Sigma_{p p / u}^{-1}$, the oblique projection is expressed as $\Pi p(t)=\mathcal{O} x(t)$, where the state vector is now defined to be the $n \times 1$ vector

$$
x(t)=\mathcal{C} \Sigma_{p p / u}^{-1} \mathbf{p}(t)=\tilde{\Sigma}^{1 / 2} V^{T} L_{p}^{-1} \mathbf{p}(t)
$$

Thus in terms of $x(t)$, the optimal predictor $\hat{\mathbf{f}}(t)$ of (1) is expressed as

$$
\hat{\mathbf{f}}(t)=\mathcal{O} x(t)+\Phi \mathbf{u}(t)
$$

where $\Phi$ is a causal operator.

\section{Innovation Model}

A forward innovation model is obtained by using the state vector $x(t)$ of $(8)$ and the optimal predictor $\hat{\mathbf{f}}(t)$ of (9). Since, from Theorem $2, \Phi$ is block lower-triangular, the first $p$ rows of (9) involves the one-step predictor of $y(t)$ based on $\mathbf{p}(t) \vee u(t)$ :

$$
\hat{y}(t):=(y(t) / \mathbf{p}(t) \vee u(t))=C x(t)+D u(t)
$$

where $C$ and $D$ are $p \times n$ and $p \times m$ constant matrices, respectively. Define the prediction error by $e(t):=$ $y(t)-\hat{y}(t)$. Then we get the output equation

$$
y(t)=C x(t)+D u(t)+e(t)
$$


Consider the dynamics of the state vector $x(t)$. Let

$$
w(t):=x(t+1)-(x(t+1) / x(t) \vee u(t))
$$

Then there exist $n \times n$ and $n \times m$ constant matrices $A$ and $B$ such that

$$
(x(t+1) / x(t) \vee u(t))=A x(t)+B u(t)
$$

Thus we have the state equation

$$
x(t+1)=A x(t)+B u(t)+w(t)
$$

It can be shown (Katayama and Picci, 1997) that the prediction error $w(t)$ is a function of $e(t)$, and hence there exists $K$ such that $w(t)=K e(t)$.

Theorem 3. Suppose that there is no feedback from the output $y(t)$ to the input $u(t)$. We assume that $\operatorname{rank} \Sigma_{f p / u}=n$. Then in terms of a state vector $x(t)$ of (8), we have a stochastic realization of the form

$$
\begin{gathered}
x(t+1)=A x(t)+B u(t)+K e(t) \\
y(t)=C x(t)+D u(t)+e(t)
\end{gathered}
$$

We note that the system of (11), (12) is the innovation form derived from the Kalman filter with the exogenous input $u(t)$.

\section{Subspace Identification Algo- rithm}

Consider a constant linear system with the input $u(t) \in$ $\mathbf{R}^{m}$ and the output $y(t) \in \mathbf{R}^{p}$. Suppose that finite input-output data $u(t), y(t)$ for $t=0,1, \cdots, N+2 k-2$ are given with $k>0$ and $N$ sufficiently large. We assume that $u(t), y(t)$ are jointly quasi-stationary processes with means zero and finite covariance matrices (Ljung, 1987). We define the block Hankel matrix

$U_{0 \mid k-1}:=\left[\begin{array}{cccc}u(0) & u(1) & \cdots & u(N-1) \\ u(1) & u(2) & \cdots & u(N) \\ \vdots & \vdots & & \vdots \\ u(k-1) & u(k) & \cdots & u(N+k-2)\end{array}\right] \in \mathbf{R}^{k m \times N}$

The block Hankel matrix $Y_{0 \mid k-1} \in \mathbf{R}^{k p \times N}$ is defined similarly. We also define the matrix formed by the state vector as

$$
X_{k}=[x(k) x(k+1) \cdots x(N+k-1)] \in \mathbf{R}^{n \times N}
$$

Let the present time be $k$. Then the past is $[0, k-1]$ and the future is $[k, 2 k-1]$. The integer $k$ should be chosen so that $k>n$, where $n$ is the dimension of the state vector to be determined. The future inputs and the future outputs are defined by $U_{k \mid 2 k-1} \in \mathbf{R}^{k m \times N}$ and $Y_{k \mid 2 k-1} \in \mathbf{R}^{k p \times N}$, respectively. Also, the past inputs-outputs is defined by $P_{0 \mid k-1}=\left[\begin{array}{c}U_{0 \mid k-1} \\ Y_{0 \mid k-1}\end{array}\right] \in$
$\mathbf{R}^{k(m+p) \times N}$. We assume that the input is persistently exciting (PE), so that $U_{0 \mid 2 k-1}$ has row rank $2 k m$.

Consider the following LQ factorization

$$
\begin{aligned}
& \frac{1}{\sqrt{N}}\left[\begin{array}{c}
U_{k \mid 2 k-1} \\
P_{0 \mid k-1} \\
Y_{k \mid 2 k-1}
\end{array}\right]=\left[\begin{array}{ccc}
L_{11} & 0 & 0 \\
L_{21} & L_{22} & 0 \\
L_{31} & L_{32} & L_{33}
\end{array}\right]\left[\begin{array}{c}
Q_{1}^{T} \\
Q_{2}^{T} \\
Q_{3}^{T}
\end{array}\right] \\
&:=L Q^{T}
\end{aligned}
$$

where $L_{11}, L_{22}$ and $L_{33}$ are lower triangular matrices and $Q_{i}$ 's are orthogonal matrices. Then we have

$$
\begin{aligned}
\hat{\Sigma}_{u p f} & :=\left[\begin{array}{ccc}
\hat{\Sigma}_{u u} & \hat{\Sigma}_{u p} & \hat{\Sigma}_{u f} \\
\hat{\Sigma}_{p u} & \hat{\Sigma}_{p p} & \hat{\Sigma}_{p f} \\
\hat{\Sigma}_{f u} & \hat{\Sigma}_{f p} & \hat{\Sigma}_{f f}
\end{array}\right] \\
& =\frac{1}{N}\left[\begin{array}{c}
U_{k \mid 2 k-1} \\
P_{0 \mid k-1} \\
Y_{k \mid 2 k-1}
\end{array}\right]\left[\begin{array}{c}
U_{k \mid 2 k-1} \\
P_{0 \mid k-1} \\
Y_{k \mid 2 k-1}
\end{array}\right]^{T}=L L^{T}
\end{aligned}
$$

Since $\hat{\Sigma}_{u u}$ is nonsingular, we have

$$
\begin{aligned}
\hat{\Sigma}_{f f / u} & =\hat{\Sigma}_{f f}-\hat{\Sigma}_{f u} \hat{\Sigma}_{u u}^{-1} \hat{\Sigma}_{u f} \\
& =L_{32} L_{32}^{T}+L_{33} L_{33}^{T}
\end{aligned}
$$

Similarly,

$$
\hat{\Sigma}_{p p / u}=L_{22} L_{22}^{T}, \quad \hat{\Sigma}_{f p / u}=L_{32} L_{22}^{T}
$$

Thus we get $\hat{\Pi}=L_{32} L_{22}^{\dagger}$.

In order to obtain $\hat{\Phi}$, we rearrange the entries of the covariance matrix of (14) to get

$$
\begin{aligned}
\hat{\Sigma}_{p u f}: & =\left[\begin{array}{lll}
\hat{\Sigma}_{p p} & \hat{\Sigma}_{p u} & \hat{\Sigma}_{p f} \\
\hat{\Sigma}_{u p} & \hat{\Sigma}_{u u} & \hat{\Sigma}_{u f} \\
\hat{\Sigma}_{f p} & \hat{\Sigma}_{f u} & \hat{\Sigma}_{f f}
\end{array}\right] \\
= & {\left[\begin{array}{ccc}
R_{11} & 0 & 0 \\
R_{21} & R_{22} & 0 \\
R_{31} & R_{32} & R_{33}
\end{array}\right]\left[\begin{array}{ccc}
R_{11}^{T} & R_{21}^{T} & R_{31}^{T} \\
0 & R_{22}^{T} & R_{32}^{T} \\
0 & 0 & R_{33}^{T}
\end{array}\right] }
\end{aligned}
$$

Hence we have

$$
\begin{gathered}
\hat{\Sigma}_{f f \mid p}=R_{32} R_{32}^{T}+R_{33} R_{33}^{T} \\
\hat{\Sigma}_{u u \mid p}=R_{22} R_{22}^{T}, \quad \hat{\Sigma}_{f u \mid p}=R_{32} R_{22}^{T}
\end{gathered}
$$

Therefore, from (3) we get

$$
\hat{\Phi}=R_{32} R_{22}^{\dagger}
$$

Computation of $A, B, C, D$

Suppose that we have the estimates of conditional covariance matrices $\hat{\Sigma}_{f f / u}, \hat{\Sigma}_{p p / u}, \hat{\Sigma}_{f p / u}$, etc.

Step 1: Compute the SVD of the normalized covariance matrix

$$
\hat{\Sigma}_{f f / u}^{-1 / 2} \hat{\Sigma}_{f p / u} \hat{\Sigma}_{p p / u}^{-1 / 2}=U S V^{T}
$$


Thus we have

$$
\hat{\Sigma}_{f p / u}=U_{f} S_{f}^{1 / 2} U_{f}^{T} U S V^{T} U_{p} S_{p}^{1 / 2} U_{p}^{T}
$$

where the dimension $n$ is estimated by $\operatorname{dim} S$.

Step 2: Set the extended observability matrix as

$$
\hat{\mathcal{O}}_{k}=U_{f} S_{f}^{1 / 2} U_{f}^{T} U S^{1 / 2}, \quad \hat{\mathcal{C}}_{k}=S^{1 / 2} V^{T} U_{p} S_{p}^{1 / 2} U_{p}^{T}
$$

Step 3: Compute $\hat{A}$ and $\hat{C}$ by

$$
\begin{gathered}
\hat{C}=\hat{\mathcal{O}}_{k}(1: p, 1: n) \\
\hat{A}=\hat{\mathcal{O}}_{k}(1:(k-1) p, 1: n)^{\dagger} \hat{\mathcal{O}}_{k}(p+1: k p, 1: n)
\end{gathered}
$$

Step 4: Compute $\hat{B}, \hat{D}$ from $\hat{\Phi}$ of (15) by using the technique in MOESP (Verhaegen and Dewilde 1992).

\section{Constraint Least Squares Method}

\section{Kronecker Products}

Let $A$ be an $m \times n$ matrix with $A=\left(a_{1}, \cdots, a_{n}\right)$, where $a_{i} \in \mathbf{R}^{n}, i=1, \cdots, n$. Then the $m n \times 1$ stacked vector $\operatorname{vec}(A)$ is defined as

$$
\operatorname{vec}(A)=\left[\begin{array}{c}
a_{1} \\
\vdots \\
a_{n}
\end{array}\right]
$$

Moreover, we define the Kronecker product $A \otimes B$ by

$$
A \otimes B=\left[\begin{array}{ccc}
a_{11} B & \cdots & a_{1 n} B \\
\vdots & \ddots & \vdots \\
a_{m 1} B & \cdots & a_{m n} B
\end{array}\right]
$$

Then for $A \in \mathbf{R}^{m \times n}$ and $B \in \mathbf{R}^{n \times l}$, $\operatorname{vec}(A B) \in \mathbf{R}^{m l}$ is expressed as

$$
\operatorname{vec}(A B)=\left(I_{l} \otimes A\right) \operatorname{vec}(B)=\left(B^{T} \otimes I_{m}\right) \operatorname{vec}(A)
$$

\section{Derivation of Algorithm}

According to Peternell et al.(1996), we derive a method of estimating $\Theta:=\left[\begin{array}{ll}\Pi & \Phi\end{array}\right]$ by (2) under the constraint that $\Phi$ is a lower-triangular Toeplitz. This leads to a constraint least-squares (CLS) method. It follows from (1) that the regression equation has the form

$$
\mathbf{f}(t)=\left[\begin{array}{ll}
\Pi & \Phi
\end{array}\right]\left[\begin{array}{l}
\mathbf{p}(t) \\
\mathbf{u}(t)
\end{array}\right]+\mathbf{v}(t)
$$

where $\mathbf{v}(t)$ is the residual. For finite data case, we get

$$
Y_{k \mid 2 k-1}=\left[\begin{array}{ll}
\Pi & \Phi
\end{array}\right]\left[\begin{array}{c}
P_{0 \mid k-1} \\
U_{k \mid 2 k-1}
\end{array}\right]+V_{k \mid 2 k-1}
$$

Vectorizing the above equation,

$$
\operatorname{vec}\left(Y_{k \mid 2 k-1}\right)=\phi^{T} \operatorname{vec}(\Theta)+\operatorname{vec}\left(V_{k \mid 2 k-1}\right)
$$

where $\Theta=\left[\begin{array}{ll}\Pi & \Phi\end{array}\right]$, and

$$
\phi^{T}=\left[\begin{array}{ll}
P_{0 \mid k-1}^{T} & U_{k \mid 2 k-1}^{T}
\end{array}\right] \otimes I_{k p}
$$

It can be shown (Peternell et al., 1996) that there exists $N_{22}$ with entries equal to zero or one such that

$$
N_{22} \operatorname{vec}\left(g_{0}, \cdots, g_{k-1}\right)=\operatorname{vec}\left[\begin{array}{cccc}
g_{0} & & & \\
g_{1} & g_{0} & & \\
\vdots & \vdots & \ddots & \\
g_{k-1} & g_{k-2} & \cdots & g_{0}
\end{array}\right]
$$

Define $N=\left[\begin{array}{cc}I & 0 \\ 0 & N_{22}\end{array}\right]$. Then we get

$$
\operatorname{vec}(\Theta)=N\left[\begin{array}{l}
\operatorname{vec}(\Pi) \\
\operatorname{vec}\left(g_{0}, \cdots, g_{k-1}\right)
\end{array}\right]:=N \theta
$$

It thus follows from (15) that

$$
\operatorname{vec}\left(Y_{k \mid 2 k-1}\right)=\phi_{k}^{T} N \theta+\operatorname{vec}\left(V_{k \mid 2 k-1}\right)
$$

Therefore the CLS estimate of $\Theta$ is given by

$$
\operatorname{vec}(\hat{\Theta})=N\left(N^{T} \phi_{k} \phi_{k}^{T} N\right)^{-1} N^{T} \phi_{k} \operatorname{vec}\left(Y_{k \mid 2 k-1}\right)
$$

For the algorithm based on CLS, we need some modifications of the CCA based method as follows.

Step 1' Given II, compute the SVD of a normalized covariance matrix

$$
\hat{\Sigma}_{f f / u}^{-1 / 2} \hat{\Pi} \hat{\Sigma}_{p p / u}^{1 / 2}=U S V^{T}
$$

to get

$$
\hat{\Sigma}_{f p / u}=U_{f} S_{f}^{1 / 2} U_{f} U S V^{T} U_{p} S_{p}^{-1 / 2} U_{p}^{T}
$$

We compute the state vector by

$$
\hat{X}_{k}=\hat{\mathcal{C}}_{k} \hat{\Sigma}_{p p / u}^{-1} P_{0 \mid k-1}
$$

and define

$$
\begin{aligned}
& Y=[y(k) y(k+1) \cdots y(N+k-1)] \\
& U=[u(k) u(k+1) \cdots u(N+k-1)]
\end{aligned}
$$

Then $\hat{A}, \hat{B}, \hat{C}$ and $\hat{D}$ are are computed by

$$
\left[\begin{array}{cc}
\hat{A} & \hat{B} \\
\hat{C} & \hat{D}
\end{array}\right]=\left[\begin{array}{c}
\hat{X}_{k+1} \\
Y
\end{array}\right]\left[\begin{array}{ll}
\hat{X}_{k}^{T} & U^{T}
\end{array}\right]\left[\begin{array}{cc}
\hat{X}_{k} \hat{X}_{k}^{T} & \hat{X}_{k} U^{T} \\
U \hat{X}_{k}^{T} & U U^{T}
\end{array}\right]^{-1}
$$

where $\hat{X}_{k+1}$ is the shifted version of $\hat{X}_{k}$. 


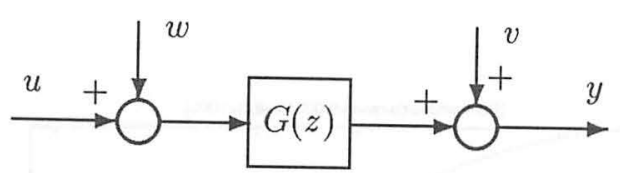

Fig. 1 The plant model

\section{Simulation Results}

Some results of computer simulations are presented to show the performance of the stochastic subspace identification algorithms derived in Sections 3 and 4. We consider a fifth-order SISO system (Viberg, 1995) shown in Fig. 1, where $u(t)$ is the input, and $w(t)$ and $v(t)$ are white noises with mean zeros. The transfer function is given by $G(z)=B(z) / A(z)$, where

$$
\begin{gathered}
B(z)=0.0275 z^{-4}+0.551 z^{-5} \\
A(z)=1-2.3443 z^{-1}+3.081 z^{-2}-2.5274 z^{-3} \\
+1.2415 z^{-4}-0.3686 z^{-5}
\end{gathered}
$$

The $G(z)$ has a zero at $z=-2$ and poles at $z=0.9$, $0.8 e^{ \pm j}, 0.8 e^{ \pm 1.2 j}$. For $N=1000$ samples of inputoutput data, we performed 100 independent runs.

\section{Example 1 (White noise input)}

In this case, the input $u(t)$ is a zero mean white Gaussian noise with variance $\sigma_{u}^{2}=1$. And the variances of noises $w(t)$ and $v(t)$ are $\sigma_{w}^{2}=0.1, \sigma_{v}^{2}=0.03$. In Figs. 2 and 3 , the pole estimates by the CCA and the CLS methods for 100 different runs are depicted, where $k=8$. Fig. 4 shows the performance of the estimation of parameters of $A(z), B(z)$ due to MOESP, CCA and CLS methods, where

$$
E=\frac{1}{100} \sum_{i=1}^{100}\left(\sum_{j=1}^{10}\left(\theta_{j}-\hat{\theta}_{j}\right)^{2}\right)
$$

with $N=200,400,1000,2000$.

\section{Example 2 (Sinusoidal input)}

To compare the effect of input signals, the input is chosen as

$$
u(t)=U_{0} \sum_{i=1}^{10} \sin \left(\omega_{i} t\right)
$$

where the frequencies $\omega_{i}$ are and where $U_{0}$ is adjusted so that we have $\sigma_{u}^{2}=1$. Since the sinusoid with 10 different frequencies is PE of order 20 (Ljung, 1987), and since it is assumed that $\operatorname{rank} U_{0 \mid 2 k-1}=2 k$, we have $k \leq 10$. The noise variances are chosen as $\sigma_{w}^{2}=\sigma_{v}^{2}=0.0001$. The results are depicted in Figs. 5 and 6 , where $k=8$. Fig. 7 also shows the performance of estimation of parameters.

\section{Conclusions}

Simulation results show that the performance of three different subspace methods (CCA, CLS and MOESP) is comparable for the white noise input. For the sinusoidal inputs, however, the performance of three methods is considerably different in that that the CLS based method gives best results in the present simulations.

\section{References}

[1] Golub, G. H. and C. R. Van Loan (1989). Matrix Computations (2nd ed.). The Johns Hopkins Univ. Press.

[2] Katayama, T., and G. Picci (1997). An Approach to Realization of Stochastic Systems with Exogenous Input, Preprints of 11th IFAC Symposium on System Identification, Kitakyushu, pp. 1107-1112.

[3] Larimore, W. E. (1990). Canonical Variate Analysis in Identification, Filtering, and Adaptive Control. In Proc. 29th IEEE Conf. Decision \& Control, Honolulu, 596-604.

[4] Lindquist, A. and G. Picci (1991). A Geometric Approach to Modelling and Estimation of Linear Stochastic Systems. $J$. Math. Systems, Estimation \& Control, 1, 241-333.

[5] Lindquist, A. and G. Picci (1996). Canonical Correlation Analysis, Approximate Covariance Extension, and Identification of Stationary Time Series. Automatica, 32, 709-733.

[6] Ljung, L. (1987). System Identification - Theory for User. Prentice-Hall.

[7] Moonen, M., B. De Moor and J. Vandewalle (1989). On- and Off-Line Identification of Linear State-Space Models, Int. J. Control, 49, 219-232.

[8] Moonen, M. and J. Vandewalle (1990). QSVD Approach to On- and Off-Line State-Space Identification. Int. J. Control, 51, 1133-1146.

[9] Peternell, K., W. Scherrer and M. Deistler (1996). Statistical Analysis of Novel Subspace Identification Methods. Signal Processing, 52, 161-177.

[10] Picci, G. and T. Katayama (1996). Stochastic Realization with Exogenous Inputs and "Subspace Methods" Identification. Signal Processing, 52, 145-160.

[11] Van Overschee, P. and B. De Moor (1993). Subspace Algorithms for the Stochastic Identification Problem. Automatica, 29, 649-660.

[12] Van Overschee, P. and B. De Moor (1994). N4SID - Subspace Algorithms for the Identification of Combined Deterministic - Stochastic Systems. Automatica, 30, 75-93.

[13] Van Overschee, P. and B. De Moor (1996). Subspace Identification for Linear Systems. Kluwer Academic Publications.

[14] Verhaegen, M. and P. Dewilde (1992). Subspace Model Identification, Part 1. The Output-Error State-Space Model Identification Class of Algorithms; Part 2. Analysis of the Elementary Output-Error State-Space Model Identification Algorithm. Int. J. Control, 56, 1187-1210 \& 1211-1241.

[15] Verhaegen, M. (1994). Identification of the Deterministic Part of MIMO State Space Models given in Innovations Form from Input-Output Data. Automatica, 30, 61-74.

[16] Viberg, M. (1995). Subspace-based Methods for the Identification of Linear Time-invariant Systems. Automatica, 31, 1835-1851 


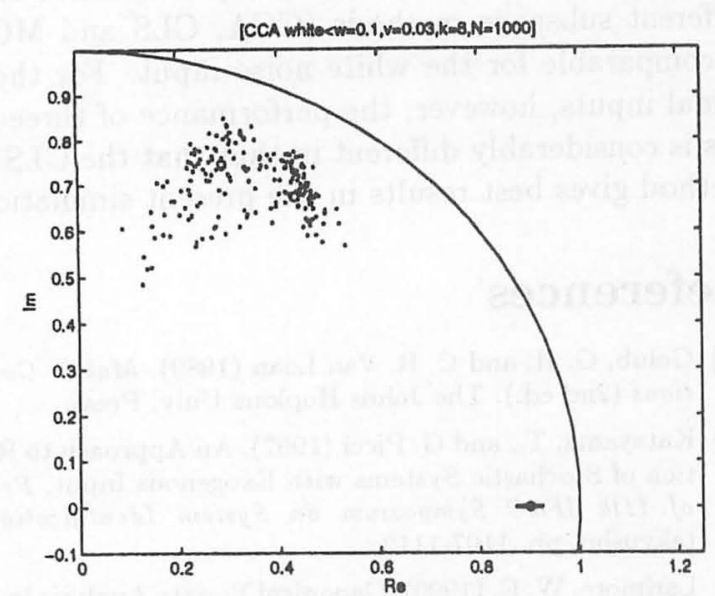

Fig. 2 The CCA method: white noise input

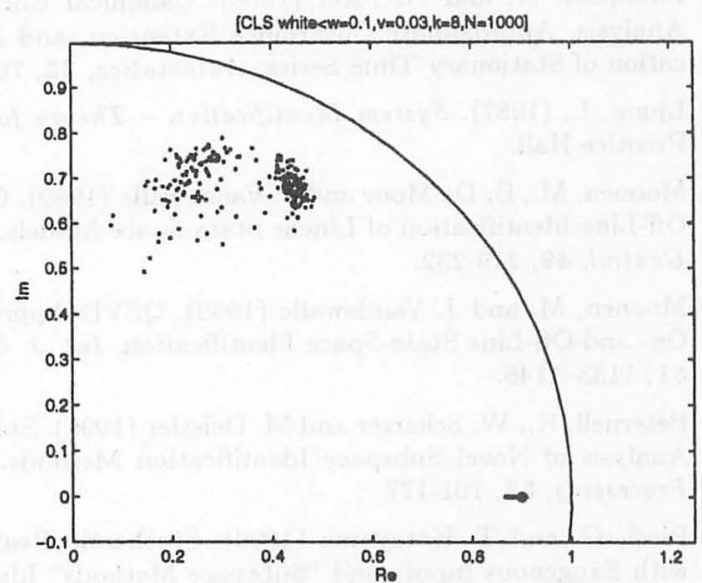

Fig. 3 The CLS method: white noise input

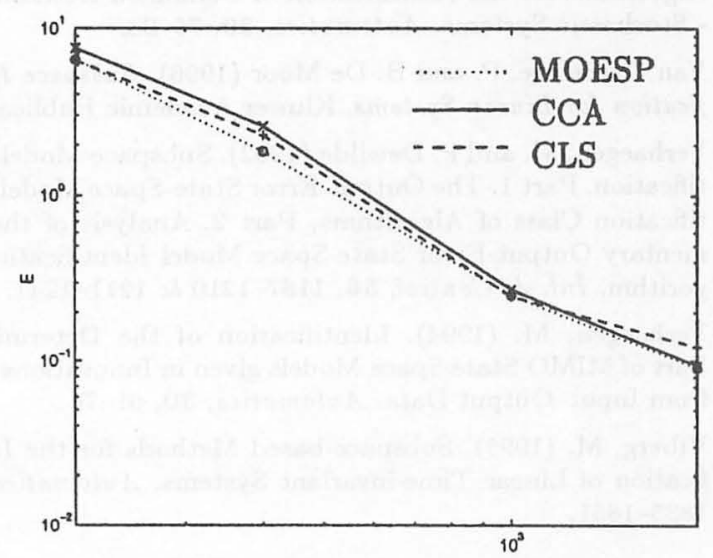

Fig. 4 Simulation result with white noise input

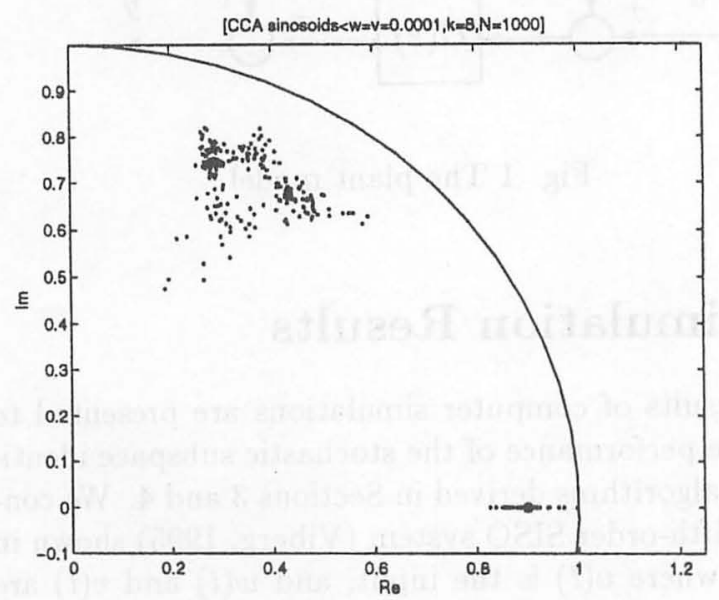

Fig. 5 The CCA method: sinosoidal input

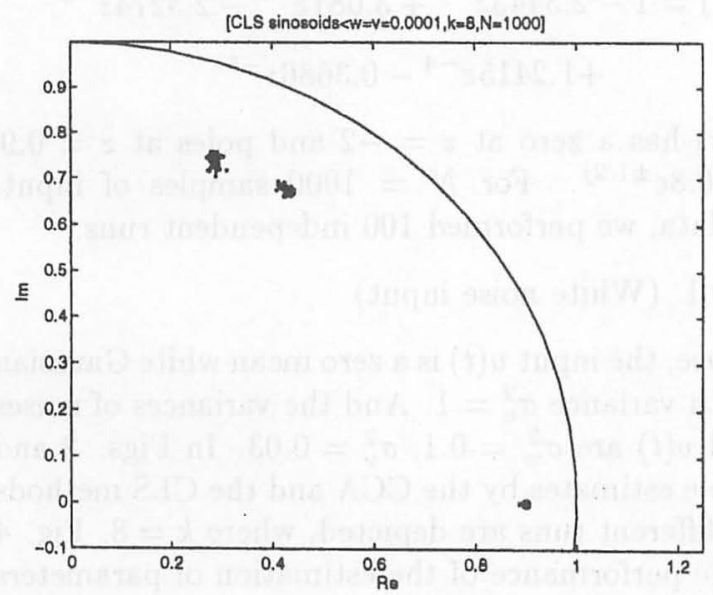

Fig. 6 The CLS method: sinosoidal input

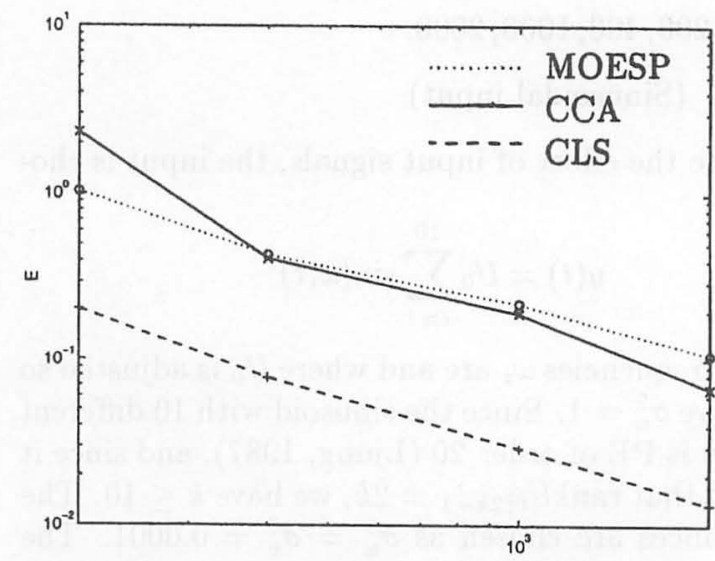

Fig. 7 Simulation result with sinosoidal input 